\title{
Type Ia Supernovae: Mechanisms and Nucleosynthesis
}

\author{
By K. NOMOTO, ${ }^{1}$ H. YAMAOKA, ${ }^{2}$ T. SHIGEYAMA, ${ }^{1}$ \\ AND K. I W A MO T O \\ ${ }^{1}$ University of Tokyo, Bunkyo-ku, Tokyo 113, Japan \\ ${ }^{2}$ Kyushu University, Fukuoka 810, Japan
}

We summarize various explosion models of Type Ia supernovae and their nucleosynthesis features for both Chandrasekhar and sub-Chandrasekhar mass white dwarf models. These models provide different predictions of the photometric and spectroscopic variations among Type Ia supernovae, which are compared with observations. Some attempts to model the peculiar SNe $1991 \mathrm{~T}$ and $1991 \mathrm{bg}$ are shown.

\section{Introduction}

Type I supernovae (SNe I) are spectroscopically defined by the absence of hydrogen in their optical spectra and further subclassified into Ia, Ib, and Ic (see, e.g., Branch et al. 1991). The early-time optical spectra of SNe Ia are characterized by the presence of a deep absorption Si II line near $6150 \AA$, and their late-time spectra are dominated by strong blends of Fe emission lines (Harkness \& Wheeler 1990). Relatively uniform light curves and spectral evolution of SNe Ia have led to the use of SNe Ia as a standard candle to determine cosmological parameters, $H_{0}$ and $q_{0}$ (Branch \& Tammann 1992).

Recent attention has been paid to variations of light curves and spectra among SNe Ia. SNe $1991 \mathrm{~T}$ and $1991 \mathrm{bg}$ have clearly revealed the presence of both spectroscopically and photometrically peculiar SNe Ia. Photometrically, maximum brightness and the decline rate of the light curve show some systematic variations, where SNe 1991T and 1991bg are situated at the two extreme ends of the brighter-slower tendency (Phillips 1993; Branch et al. 1993). Spectroscopically, the pre-maximum spectra of SNe Ia reveal a significant variation of the composition and expansion velocities of the outermost layers, whereas the post-maximum spectra are relatively uniform except for SN 1991bg.

Theoretically thermonuclear explosions of accreting white dwarfs have been considered to be the most promising models for SNe Ia (e.g., Nomoto 1986, 1994; Woosley \& Weaver 1986b). The model that the white dwarf undergoes explosion when its mass becomes close to the Chandrasekhar limit is consistent with the uniformity of SNe Ia. However, the above variations and peculiarities have challenged the Chandrasekhar mass models.

In this review, various types of thermonuclear explosion models and their nucleosynthesis features are summarized. We show some attempts to interpret the variations among SNe Ia including SN 1991T and SN 1991bg and to clarify whether these peculiar SNe Ia belong to a different subclass of SNe I or are only small variant of typical SNe Ia.

\section{Evolution of Accreting White Dwarfs}

Though the white dwarf models can account for many of the observed features of SNe Ia, the exact binary evolution that leads to SNe Ia has not been identified (e.g., Renzini 1994). The outcome of an accreting white dwarf depends on the accretion rate $\dot{M}$ and the composition ( $\mathrm{H}, \mathrm{He}, \mathrm{C}+\mathrm{O}$ ) of materials transferred from the companion star 
as well as the initial mass of the white dwarf (e.g., Nomoto 1982a; Nomoto \& Kondo 1991). Chandrasekhar mass white dwarfs could be reached for relatively high accretion rate such as $\dot{M} \approx 10^{-8}-10^{-6} M_{\odot} \mathrm{yr}^{-1}$ thanks to relatively small mass ejection after hydrogen (or helium) shell burning near the surface. Merging of double white dwarfs has been suggested to be an alternative way to form Chandrasekhar mass white dwarfs (Iben \& Tutukov 1984; Webbink 1984), though the actual merging process is poorly understood and could lead to different outcome depending on the combination of the white dwarf pair.

The explosion of sub-Chandrasekhar mass white dwarfs is a possible alternative to the above scenario. For $10^{-8} \quad M_{\odot} \mathrm{yr}^{-1} \gtrsim \dot{M} \gtrsim 10^{-9} \quad M_{\odot} \mathrm{yr}^{-1}$, the ignited helium shell flash is strong enough to initiate an off-center helium detonation (Nomoto 1982b; Woosley et al. 1986; Hashimoto et al. 1986). This leads to various types of explosions, which includes a central carbon detonation ignited by a helium detonation-induced shock wave (Livne 1990). Also a series of carbon shell flashes during merging of double white dwarfs could produce shock waves that propagate toward the central region to become strong enough to ignite a central carbon detonation because of decreasing area of the converging front (Iben 1988; Wheeler \& Harkness 1990; Shigeyama et al. 1992).

Among the various models predicted from the possible presupernova evolution, we discuss only the models which would at least satisfy the basic constraints from observations, i.e., the production of a significant amount of ${ }^{56} \mathrm{Ni}$ and intermediate mass elements such as $\mathrm{Ca}, \mathrm{S}, \mathrm{Si}, \mathrm{Mg}$, and $\mathrm{O}$. Thus the carbon detonation in the Chandrasekhar mass white dwarfs (Arnett 1969), the helium detonation in helium white dwarfs (Nomoto \& Sugimoto 1977; Woosley et al. 1986), and the He/C double detonations (Nomoto 1982b; Woosley et al. 1986; Dgani \& Livio 1990) are not discussed, since these models produce almost entirely iron peak elements. The good feature of the following models is that the white dwarf has relatively low density layers where the deflagration or detonation synthesizes the intermediate mass elements.

\section{Chandrasekhar Mass Models}

For the Chandrasekhar mass white dwarf model, carbon burning in the central region leads to thermonuclear runway. At such a high central density as $\sim 3 \times 10^{9} \mathrm{~g}$ $\mathrm{cm}^{-3}$, nuclear energy release is only $\sim 20 \%$ of the Fermi energy of degenerate electrons. Therefore, the resulting shock wave was found to be not strong enough to directly form a supersonic detonation wave (Ivanova et al. 1975; Nomoto et al. 1976). [Formation of a detonation wave depends on the temperature gradient or preheating in front of the deflagrating region (Blinnikov \& Khokhlov 1989; Barkat et al. 1990; Woosley 1990), so that studies of convective energy transport during initial thermonuclear runaway are important. Observationally, the nucleosynthesis outcome of carbon detonation, being mostly Fe (Arnett 1969), is not favored, however.]

Afterwards the flame front propagates at a subsonic speed as a deflagration wave due to heat transport across the front. Since densities in the burned layers are smaller than the overlying unburned layers, the flame front becomes Rayleigh-Taylor unstable. The resulting mixing of unburned materials into the hot region and the deformation of the burning front enhances the flame speed as simulated in 1D (Nomoto et al. 1976, 1984; Woosley \& Weaver 1986a,b; Woosley 1990) and 2D (Müller \& Arnett 1986; Livne 1993; Arnett 1994) calculations. Since the propagation of the deflagration wave is subsonic, the density it encounters in the outer layer has already decreased due to the expansion of the white dwarf. 


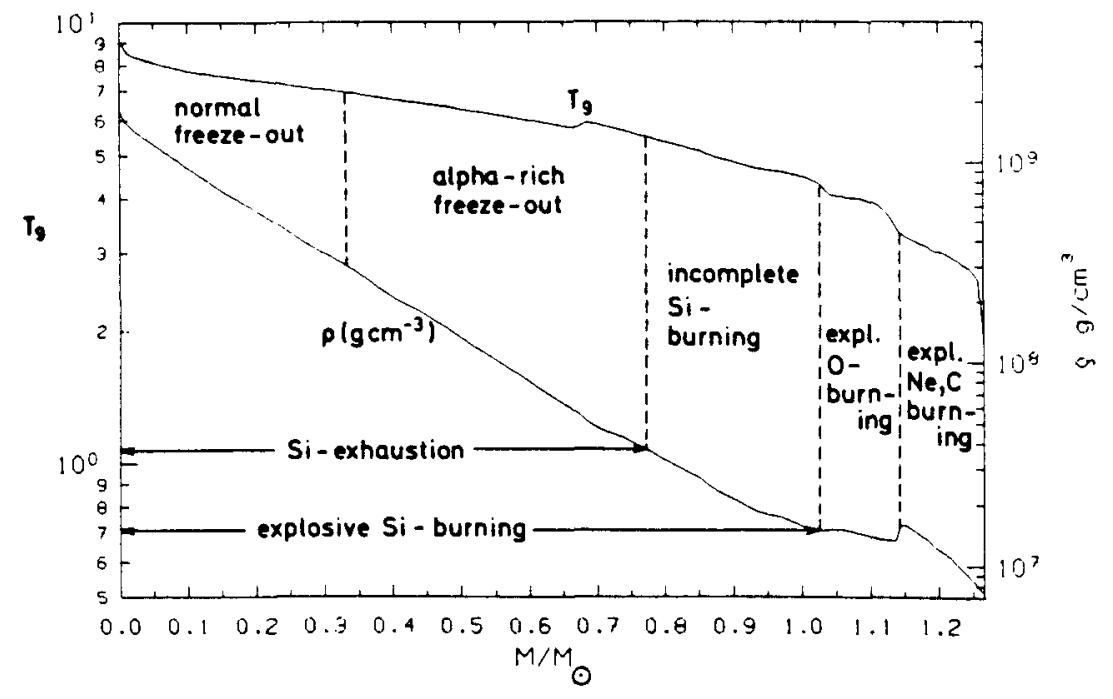

FIGURE 1. Various modes of nuclear burning as a function of peak temperatures and densities attained at the deflagration front of model W7 (Thielemann et al. 1986).

\subsection{Fast Carbon Deflagration and Late Detonation}

The carbon deflagration model W7 (Nomoto et al. 1984), whose flame speed is on the average about one-fifth of the sound speed, shows a good example of explosive nucleosynthesis as a function of the density at the flame front (Fig. 1: Thielemann et al. 1986). In the inner layer $\left(\rho>2 \times 10^{8} \mathrm{~g} \mathrm{~cm}^{-3}\right)$, materials are incinerated into iron-peak elements, mostly ${ }^{56} \mathrm{Ni}$, while in the outer lower density layers $\left(\rho \approx 5-10 \times 10^{7} \mathrm{~g} \mathrm{~cm}^{-3}\right)$, the peak temperature is too low to complete silicon burning and thus only $\mathrm{Ca}, \mathrm{Ar}, \mathrm{S}$, and $\mathrm{Si}$ are produced from oxygen burning. In the layers with $\approx 2-5 \times 10^{7} \mathrm{~g} \mathrm{~cm}^{-3}$, explosive burning of carbon and neon synthesizes $\mathrm{S}, \mathrm{Si}$, and $\mathrm{Mg}$. In the outermost layers $(\lesssim 1 \times$ $10^{7} \mathrm{~g} \mathrm{~cm}^{-3}$ ), the deflagration wave dies and $\mathrm{C}+\mathrm{O}$ remain unburned. The composition structure after freeze-out is shown as a function of $M_{r}$ and the expansion velocity $v_{\exp }$ in Figure 2. Synthetic spectra of W7 has been found to be in excellent agreement with the observed optical spectra of SN 1981B (Branch et al. 1985) and 1989B (Harkness 1991).

[Since nucleosynthesis in the above model depends on the competition between the propagation of the deflagration wave and the expansion of the white dwarf, the white dwarf model in the hydrodynamical calculation should include a realistic outer layer (or proper outer boundary conditions) owing to the subsonic nature of explosion. The outermost layer of the white dwarf models in Nomoto et al. $(1976,1984)$ is extended to the hydrogen burning shell which processes the accreting matter, thus exerting significantly larger tamping effects on the expansion of the white dwarf than in the zero-pressure boundary models. This might be the source of differences with the deflagration models by Khokhlov et al. (1993).]

In the outer layers a relatively fast deflagration induces a formation of a detonation wave. For example, the carbon deflagration model $\mathrm{C} 8$, whose propagation velocity $v_{\text {def }}$ is slightly higher than W7, has a precursor shock which is strong enough to induce a detonation in the outer layers (Nomoto et al. 1984; model F7 in Woosley \& Weaver 1986a; delayed detonation models in Khokhlov 1991a). The transition from deflagration to detonation can occur when the flame speed $v_{\text {def }}$ is accelerated to the Chapman-Jouguet velocity $v_{\mathrm{CJ}}$. The transition is more likely to occur at lower densities, because the ratio 


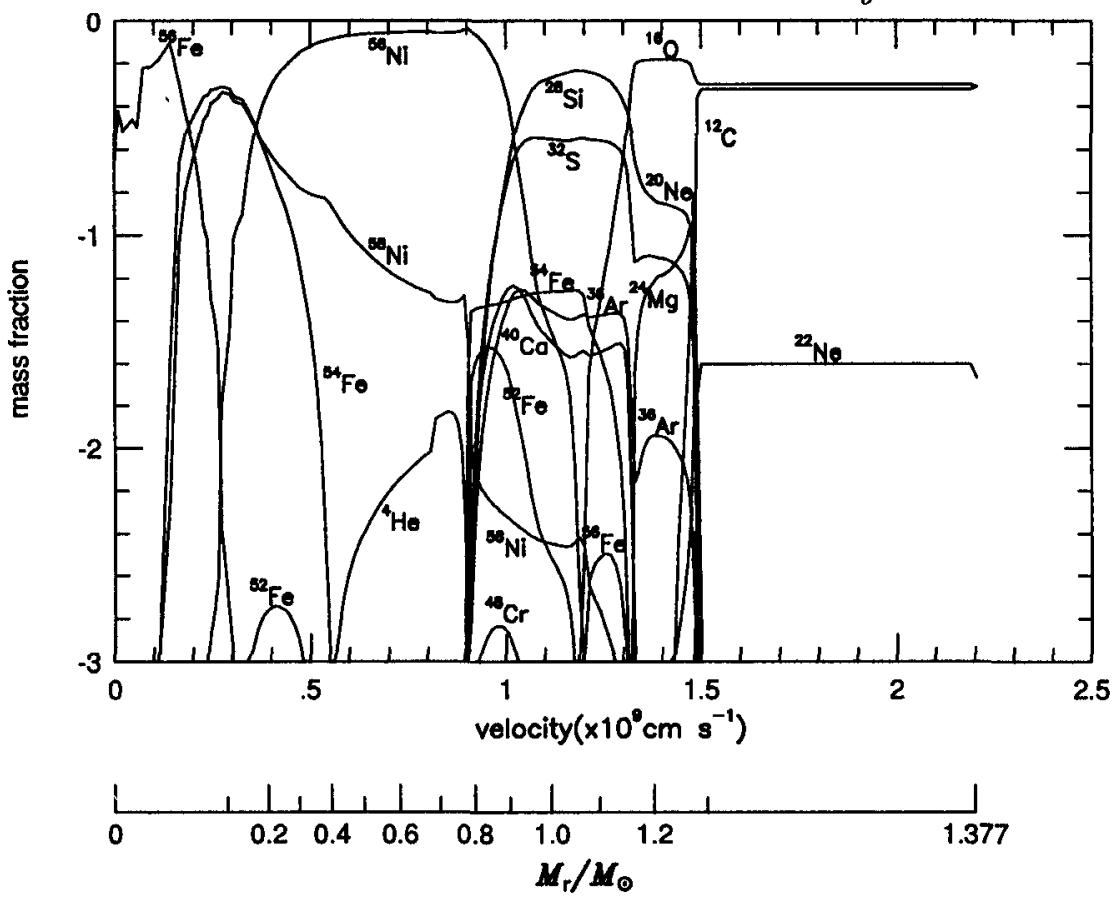

Figure 2. Composition of a carbon deflagration model W7 for Type Ia supernovae as a function of interior mass and the expansion velocity (Nomoto et al. 1984).

$v_{\mathrm{CJ}} / v_{\mathrm{s}}$ ( $v_{\mathrm{s}}$ denotes the sound velocity) is smaller due to a larger density jump across the flame front (Khokhlov 1991a), while $v_{\text {def }} / v_{\mathrm{s}}$ is larger (Nomoto et al. 1984). Since the convective deflagration front may well be quite turbulent and the acceleration of $v_{\text {def }}$ may take place in an indeterministic manner, the condition of the transition from deflagration to detonation has not been well understood (e.g., Williams 1985) and it could occur even for $v_{\text {def }}<v_{\mathrm{CJ}}$ (Khokhlov 1991a; Woosley \& Weaver 1994a).

Yamaoka et al. (1992) presented several late detonation models (W7DN, W7DT, W7DHE, and W8DT) where the fast carbon deflagration like W7 and W8 produces a central $\mathrm{Fe} / \mathrm{Co} / \mathrm{Ni}$ core and an intermediate $\mathrm{Si} / \mathrm{S} / \mathrm{Ca}$ layer and later is transformed into a detonation in the outermost layers. In these models the expansion velocities of $\mathrm{Si}$ and $\mathrm{S}$ in the outer layers well exceed $20,000 \mathrm{~km} \mathrm{~s}^{-1}$.

\subsection{Slow Deflagration and Delayed Detonation}

The outcome of carbon deflagration depends on its flame speed, which is highly uncertain. If the flame speed is much lower than in $W 7$, the hydrodynamical behavior is very different. Figure 3 shows the expansion and oscillation of the white dwarf for the propagation speed of 20 times smaller than in W7, i.e., about 1/100 of the sound speed on the average (Nomoto et al. 1976). The white dwarf expands to quench nuclear burning at stage 6 , where the total energy of the star is still negative. Then the star contracts to burn more material, though the density is too low to produce another ${ }^{56} \mathrm{Ni}$. This makes the total energy positive $\left(\sim 5 \times 10^{49} \mathrm{erg} \mathrm{s}^{-1}\right)$. Eventually the white dwarf is completely disrupted with the mass of ${ }^{56} \mathrm{Ni} M_{\mathrm{Ni}} \sim 0.15 M_{\odot}$.

Such a slow flame model with oscillation produces too small a kinetic energy of explosion to account for SN Ia. As mentioned in $\S 3.1$, however, the deflagration might induce a detonation at low density layers. In the delayed detonation model (Khokhlov 1991a; 


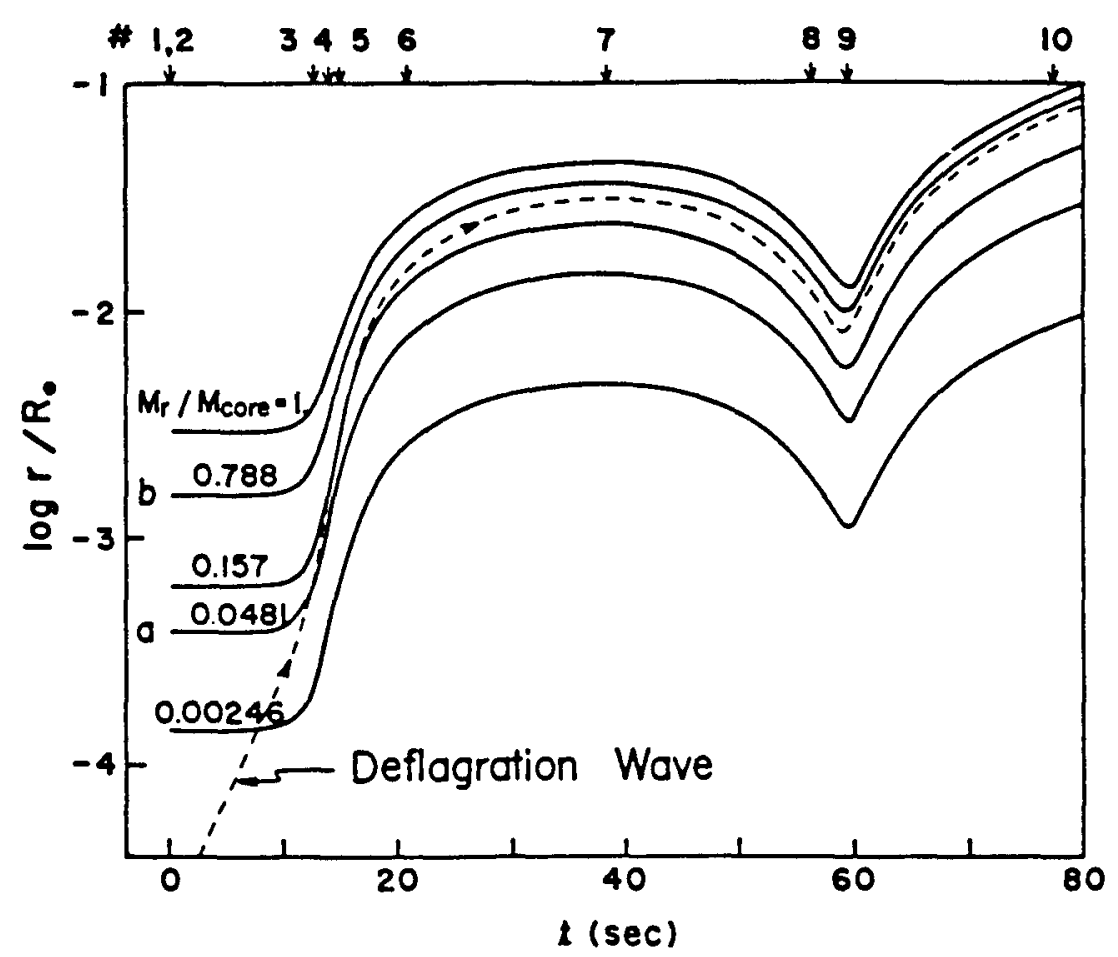

FIGURE 3. Expansion and oscillation of the white dwarf induced by a slow carbon deflagration (Nomoto et al. 1976).

Woosley \& Weaver 1994a), the deflagration wave is assumed to be transformed into detonation at a certain layer during the first expansion phase; here a quick acceleration of the flame speed to $v_{\mathrm{CJ}}$ or quite a turbulent frame is assumed at the transition. In the pulsating delayed detonation model (Khokhlov 1991b), the transition to detonation is assumed to occur near the maximum compression due to mixing and compression. In these models, a carbon detonation propagates through the layers with $\rho<10^{8} \mathrm{~g} \mathrm{~cm}^{-3}$, which is much lower than in the central carbon detonation model.

Figure 4 shows a composition structure of such a delayed detonation model against the expansion velocity and $M_{r}$, which is simulated by assuming that the transition from the deflagration to the detonation occurs when the density at $M_{r}=0.6 M_{\odot}$ has been decreased to $3 \times 10^{7} \mathrm{~g} \mathrm{~cm}^{-3}$ (Nomoto et al. 1991, 1994). Because this model includes explosive carbon burning at low densities similar to W7, the composition structure with respect to $M_{r}$ is not so different from W7 including some unburned $\mathrm{C}$ and $\mathrm{O}$, but $\mathrm{Si}$ and $\mathrm{Ca}$ layers extend to the expansion velocities higher than $15,000 \mathrm{~km} \mathrm{~s}^{-1}$.

\section{Sub-Chandrasekhar Mass Models}

As mentioned in $\S 2$, a carbon detonation in a sub-Chandrasekhar mass white dwarf may possibly be induced by off-center helium detonation (Livne 1990; Woosley \& Weaver 1994b) and by off-center carbon flashes (Shigeyama et al. 1992). Because of low central densities, nucleosynthesis in the detonation wave produces a significant amount of intermediate mass elements. This is in contrast to the carbon detonation model where the explosive carbon burning takes place at such high densities as $\gtrsim 10^{8} \mathrm{~g} \mathrm{~cm}^{-3}$ and 


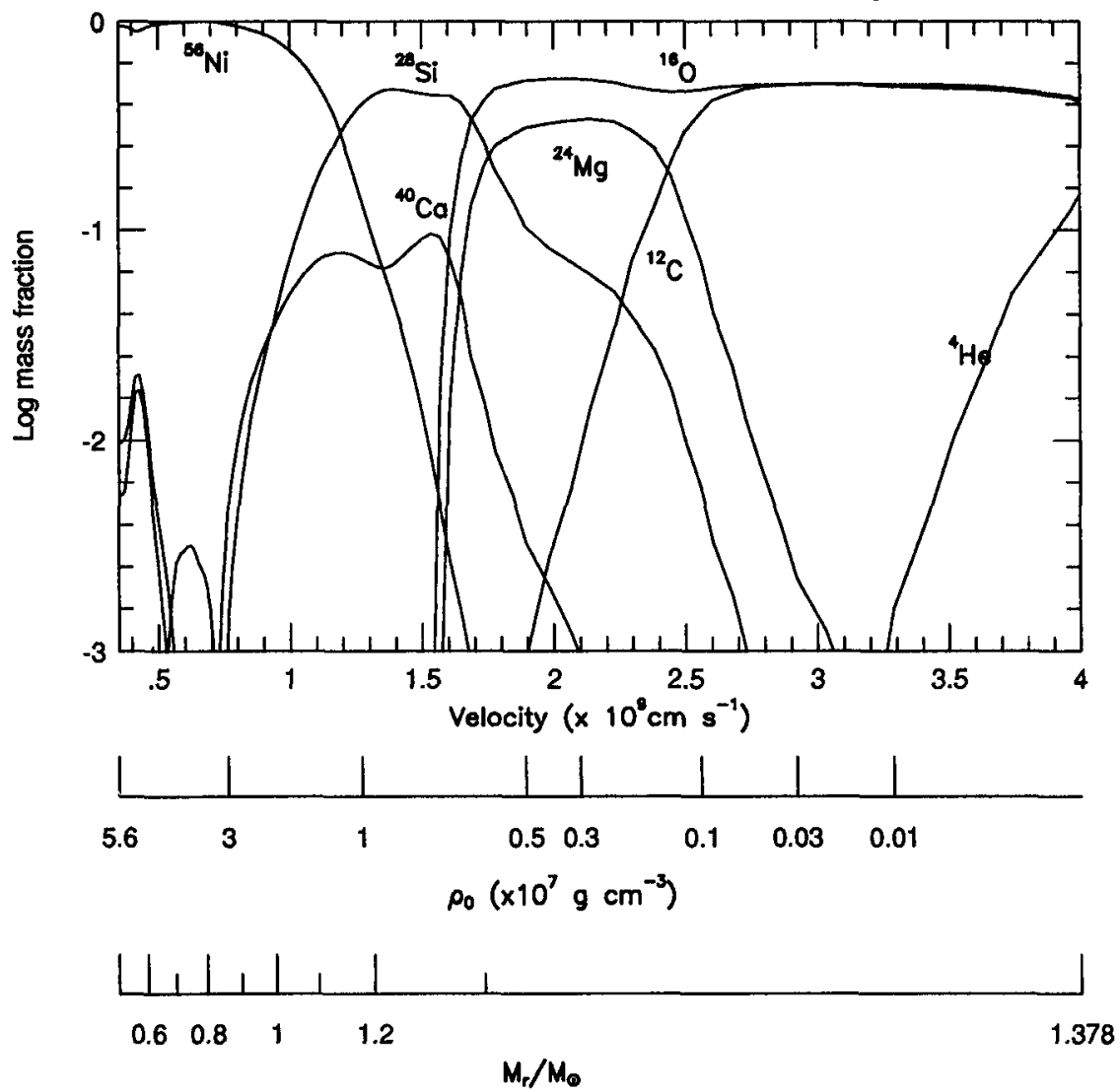

Figure 4. Composition of a delayed detonation model as a function of the expansion velocity, $M_{\mathrm{r}}$, and the initial density (Nomoto et al. 1991, 1994).

produces mostly iron-peak elements (Arnett 1969). For the carbon shell flash scenario, hydrodynamical calculations were carried out for the white dwarfs with $M=1.0-1.1$ $M_{\odot}$ and central densities of $2-5 \times 10^{7} \mathrm{~g} \mathrm{~cm}^{-3}$, where the carbon detonation produces $0.5-0.7 M_{\odot}{ }^{56} \mathrm{Ni}$ and the kinetic energy of $1.2-1.3 \times 10^{51}$ ergs (Shigeyama et al. 1992). Note that the above mass of ${ }^{56} \mathrm{Ni}$ and the explosion energy are not so different from the Chandrasekhar mass models. Figure 5 shows a composition distribution as a function of $M_{r}$ and the expansion velocity for the carbon detonation in the $1.05 M_{\odot}$ white dwarf. The outer layers are Si-rich which is similar to W7, but Si and Ca layers extend to the expansion velocities higher than $15,000 \mathrm{~km} \mathrm{~s}^{-1}$. Thus the carbon detonation models can account for the basic features of SNe Ia with a restricted range of white dwarf mass $(M \approx$ $\left.1.0-1.2 M_{\odot}\right)$.

In the helium-detonation-induced models, the off-center helium detonation produces ${ }^{56} \mathrm{Ni}$ and $\mathrm{He}$ together in the high velocity outermost layers. Because of the presence of radioactive ${ }^{56} \mathrm{Ni}$ near the surface, the spectra would be highly non-LTE. Whether the helium features excited by $\gamma$-rays appear or not would be a crucial diagnosis (Lucy 1991; Swartz et al. 1993). Examinations of the non-LTE synthetic spectra for these models are necessary. Also, since there is no oxygen in the outermost layers, the velocity of oxygen is much less than $22,000 \mathrm{~km} \mathrm{~s}^{-1}$ (e.g., $\lesssim 14,000 \mathrm{~km} \mathrm{~s}^{-1}$ in the W7DHE model; Yamaoka et al. 1992) unless some non-spherical effects operate.

If the majority of $\mathrm{SNe}$ Ia originate from the sub-Chandrasekhar mass white dwarfs 


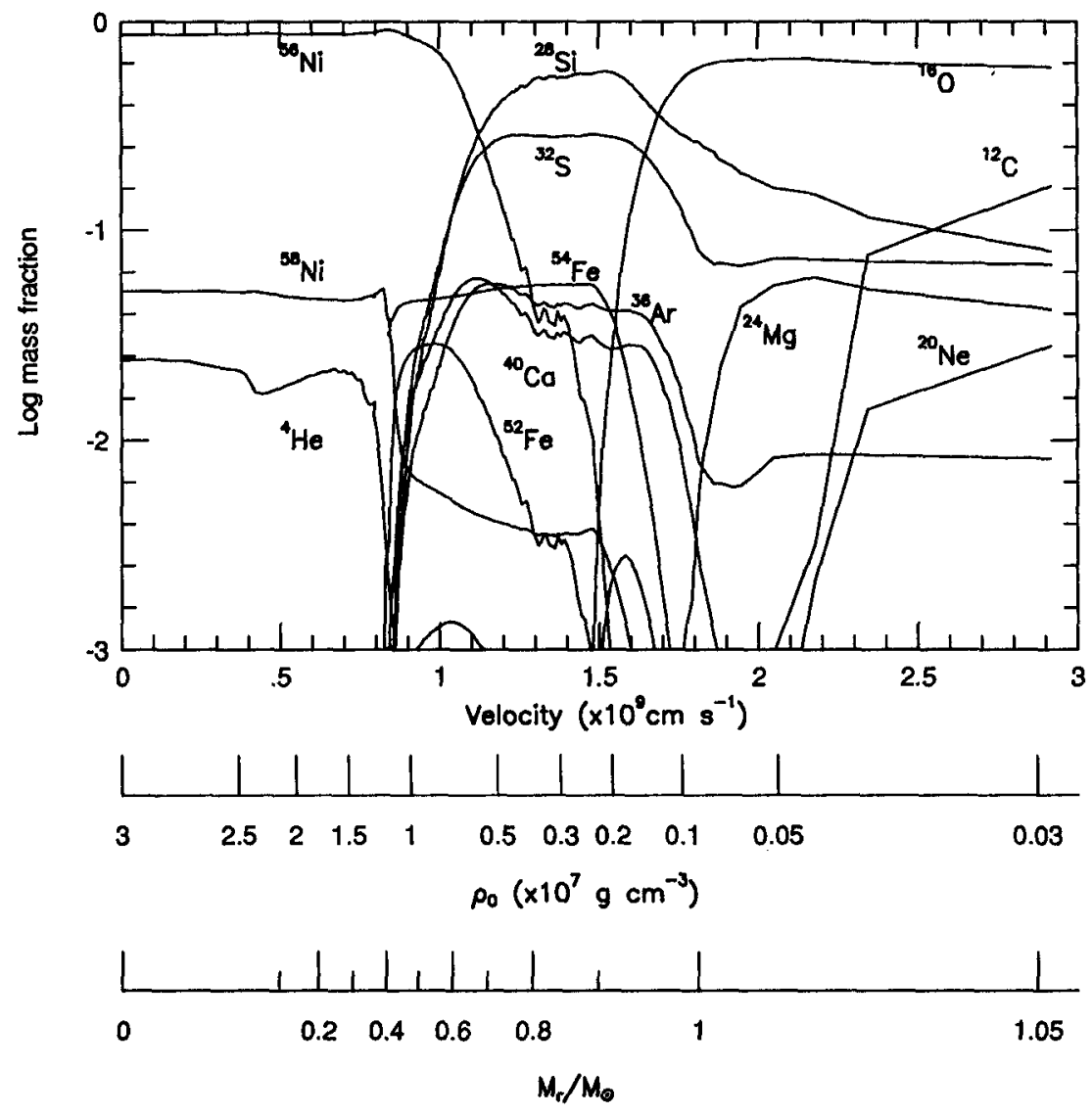

FIGURE 5. Composition of a carbon detonation model of the $1.05 M_{\odot}$ white dwarf as functions of the expansion velocity, $M_{r}$, and the initial density of the white dwarf (Shigeyama et al. 1992).

with possibly a wide range of masses, the question is why SNe Ia are quite uniform. The answer could be as follows: The initial mass of a white dwarf at its formation in a close binary system is likely to be $\sim 1.0 M_{\odot}$ rather than $\sim 0.6 M_{\odot}$ if it is formed from case $\mathrm{BB}$ binary evolution. In case $\mathrm{BB}$, a star with an initial mass of $5-8 M_{\odot}$ once becomes a helium star of $1.5-2 M_{\odot}$, which greatly expands to undergo the Roche lobe overflow when its degenerate $\mathrm{C}+\mathrm{O}$ core grows to $\sim 1 M_{\odot}$ (e.g., Sugimoto \& Nomoto 1980; Nomoto 1982c). Since the onset stage of the helium envelope expansion depends on the density contrast between the central region and the envelope, the resulting white dwarf mass $\left(\sim 1 M_{\odot}\right)$ is determined strongly by the electron degeneracy in the central region of the core, and thereby depends only weakly on the envelope mass.

\section{Peculiar Type Ia Supernovae}

\subsection{SN 1991T}

The composition structure of SN $1991 \mathrm{~T}$ is quite unique (Filippenko et al. 1992a; RuizLapuente et al. 1992; Phillips et al. 1992; Mazzali et al. 1993) consisting of (I) the outermost layer composed of $\mathrm{Ni}$ and $\mathrm{Fe}$, (II) the intermediate layer rich in $\mathrm{Si} / \mathrm{Ca}$, and (III) the central layer dominated by Fe. In other words, the Si/S/Ca-rich layer (II) 


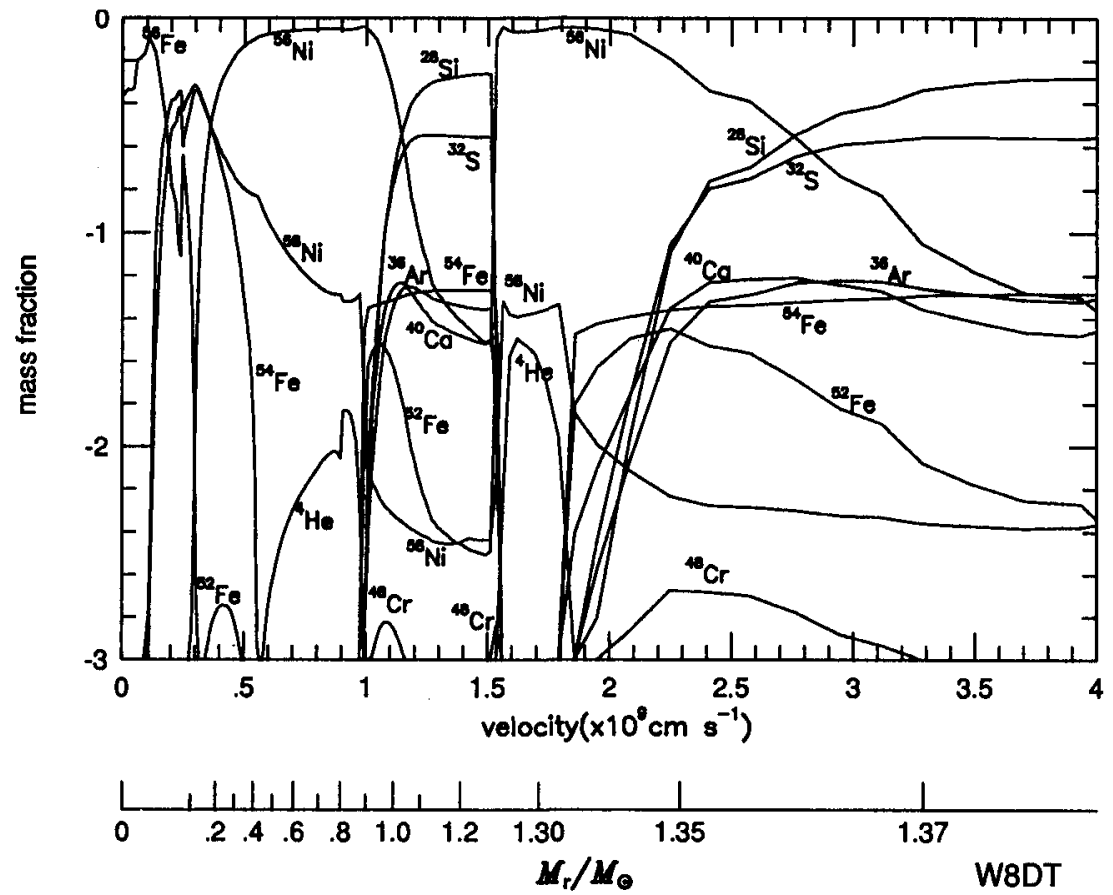

Figure 6. Composition of the late detonation model W8DT as a function of the expansion velocity and $M_{r}$, where the transition from deflagration to detonation takes place at $M_{r}=1.25$ $M_{\odot}$ where the density ahead of the flame front is $3.5 \times 10^{7} \mathrm{~g} \mathrm{~cm}^{-3}$ (Nomoto 1993).

is sandwiched by the two Fe layers (I and III). Such a composition inversion with Fe above $\mathrm{Si}$ is very different from that inferred from typical SNe Ia. On the other hand, the composition of the inner layers (II and III) is similar to those of typical SNe Ia (Filippenko et al. 1992a; Jeffery et al. 1992), though the Fe mass seems to be somewhat larger than typical SNe Ia (Mazzali 1992).

The off-center helium detonation model (Filippenko et al. 1992a) and the late detonation model (Yamaoka et al. 1992) have been suggested to account for the presence of the layer (I). The off-center helium detonation model predicts the presence of a significant amount of He together with ${ }^{56} \mathrm{Ni}$ in the layer (I), thus having the same problem of unseen He lines as discussed in $§ 4$.

Yamaoka et al. (1992) applied the late detonation model to account for not only the high velocity Si features in some SNe Ia but also the above features of SN 1991T in a unified manner. Nucleosynthesis in the detonated matter depends sensitively on the density at the transition from deflagration to detonation; for higher density, more $\mathrm{Fe}$ relative to $\mathrm{Si}$ is produced as seen from the composition structures of the late detonation models W7DN, W7DT, and W8DT (Fig. 6). In W8DT, the deflagration W8 whose flame speed is 1.2 times faster than W7 is transformed into detonation. Models W7DT and W8DT may account for the basic features of SN 1991T as seen from the good agreement between the calculated light curve for W7DT and observations in Figure 7.

\section{2. $S N 1991 b g$}

SN 1991bg is definitely dimmer than typical SNe Ia; its maximum brightness is about one fourth of SN 1957B which appeared in the same host galaxy NGC 4374 (M84). Also the decline of the light curve after maximum is significantly faster (Filippenko et al. 1992b; Leibundgut et al. 1993). These features imply that the produced ${ }^{56} \mathrm{Ni}$ is as 


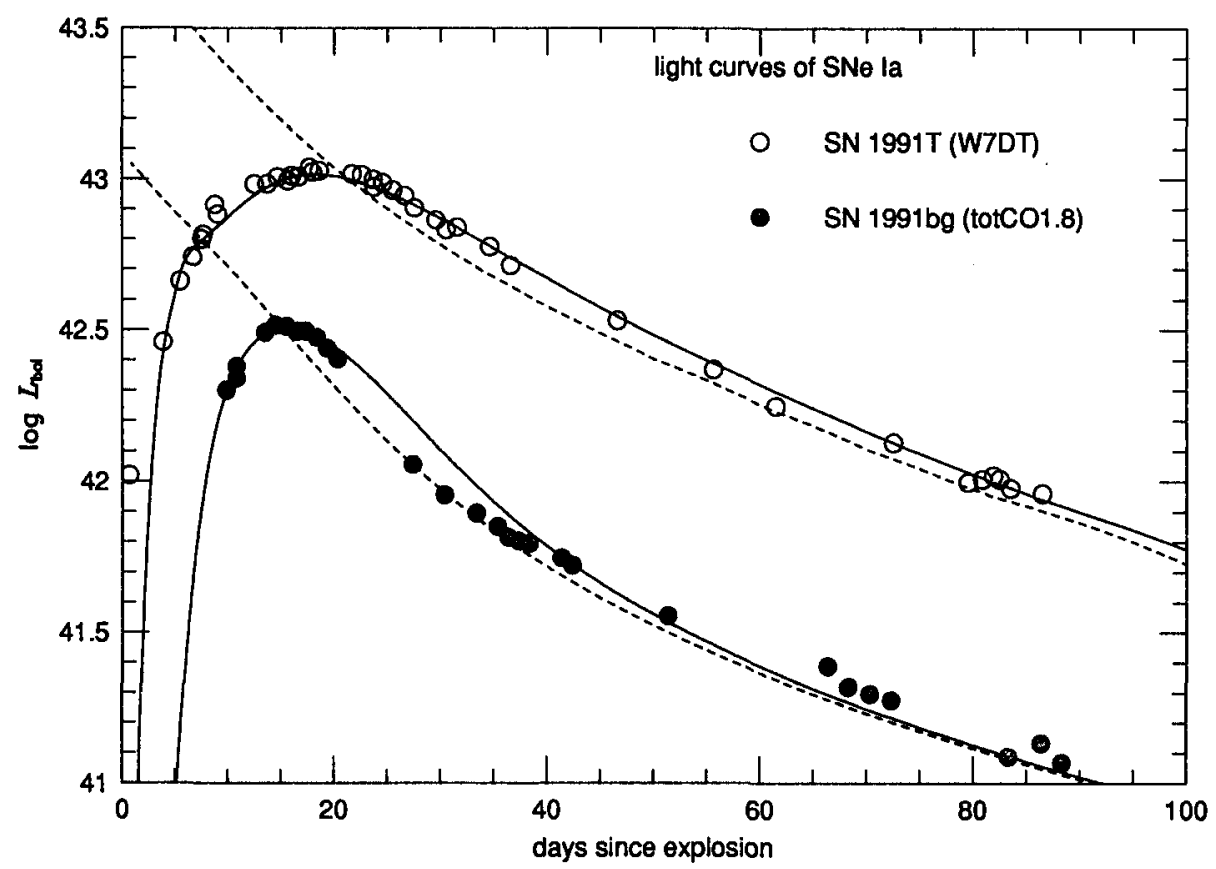

FIGURE 7. The observed visual light curves of the two peculiar SNe Ia, 1991T and 1991bg, are compared with the calculated bolometric light curves for the late detonation model W7DT and the $0.6 M_{\odot} \mathrm{C}+\mathrm{O}$ envelope model, respectively. For both models constant optical opacity $\kappa=$ $0.3 \mathrm{~cm}^{2} \mathrm{~g}^{-1}$ is assumed (Yamaoka et al. 1994b).

small as $\sim 0.15 M_{\odot}$ (Phillips 1993) and the ejected mass is also much smaller than the Chandrasekhar mass.

These features lead to the suggestion that SN 1991bg is the explosion of a white dwarf of $M \sim 0.7 M_{\odot}$, which undergoes a carbon detonation induced by an off-center helium detonation (Ruiz-Lapuente et al. 1993b; Canal \& Ruiz-Lapuente 1994). A problem with such a small mass white dwarf is that more than $\sim 0.2-0.3 M_{\odot}$ He layer should have been accreted to make the density sufficiently high $\left(\gtrsim 2-3 \times 10^{6} \mathrm{~g} \mathrm{~cm}^{-3}\right)$ at the base of the accreted layer to initiate an off-center helium detonation. A total white dwarf mass of $0.8 M_{\odot}$ of which $\sim 0.2 M_{\odot}$ is He would be necessary to initiate an off-center $\mathrm{He}$ detonation (Nomoto et al. 1994). Then the produced ${ }^{56} \mathrm{Ni}$ mass would exceed $0.3 M_{\odot}$, and so the supernova would be much brighter than SN $1991 \mathrm{bg}$.

Yamaoka et al. (1994b) have constructed a different scenario by invoking a collapse of a white dwarf in the merging of a pair of an $\mathrm{O}+\mathrm{Ne}+\mathrm{Mg}$ white dwarf and a $\mathrm{C}+\mathrm{O}$ white dwarf. When the smaller mass $\mathrm{C}+\mathrm{O}$ white dwarf fills its Roche lobe, the resultant rapid mass transfer from the $\mathrm{C}+\mathrm{O}$ white dwarf would form a thick disk around the more massive $\mathrm{O}+\mathrm{Ne}+\mathrm{Mg}$ white dwarf (Hachisu et al. 1986; Benz et al. 1990; Mochkovitch \& Livio 1990). After the cooling of the outer disk, the $\mathrm{O}+\mathrm{Ne}+\mathrm{Mg}$ white dwarf would collapse due to electron capture (Miyaji et al. 1980; Nomoto \& Kondo 1991). [A recent update of electron capture rates (Takahara et al. 1989) does not alter the above result of collapse (Hashimoto et al. 1993).] The collapsing white dwarf is surrounded by the circumstellar envelope composed of original $\mathrm{C}+\mathrm{O}$ white dwarf matter. If the envelope has become sufficiently cool and as dense as the mantle around the iron core of Type II supernovae (SNe II), the shock wave from the collapsing white dwarf propagates through the envelope to produce some ${ }^{56} \mathrm{Ni}$ and other elements as in SNe II. 


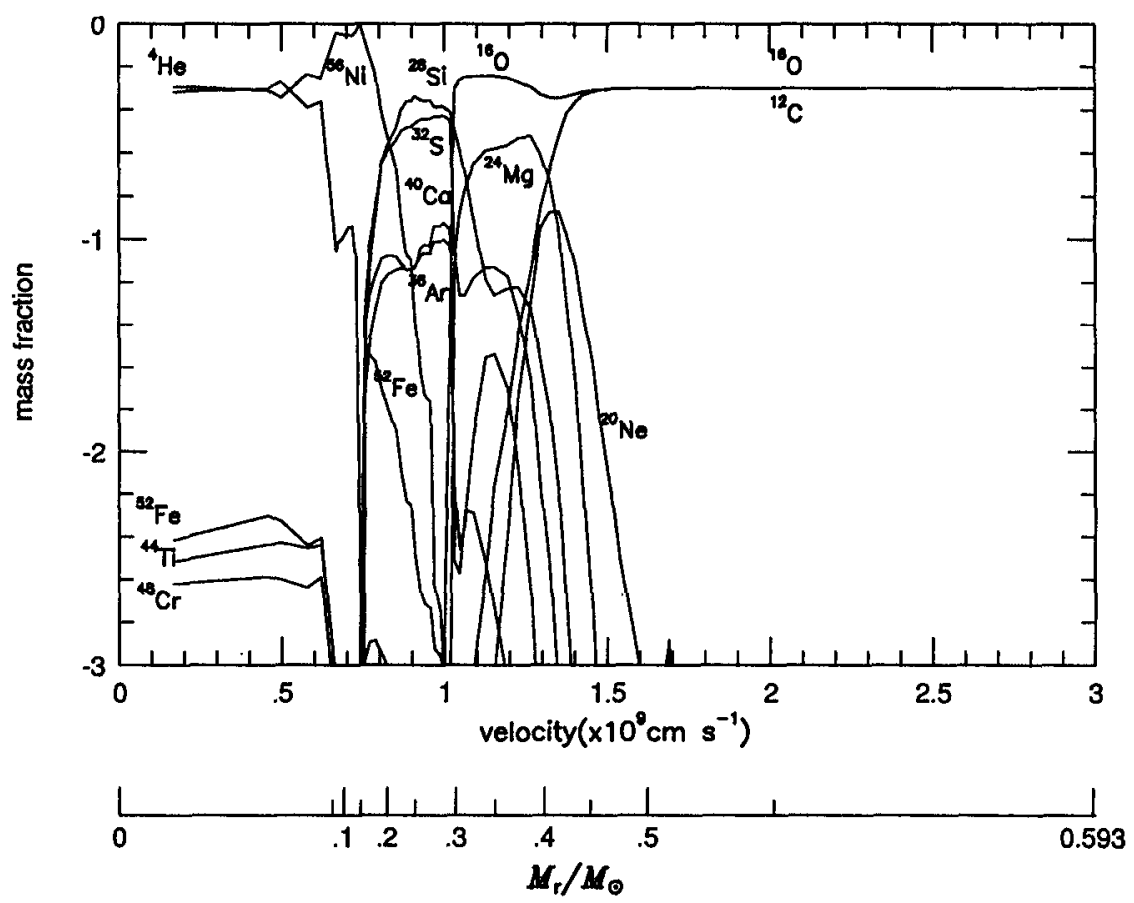

Figure 8. Composition of the $0.6 M_{\odot}$ ejecta as a function of the expansion velocity and $M_{r}$ as a model for SN 1991bg. The materials are processed by the shock wave generated at the collapse of a white dwarf (Yamaoka et al. 1994b).

Figures 7 and 8 show the composition structure and the optical light curve for the exploding $0.6 M_{\odot} \mathrm{C}+\mathrm{O}$ envelope around the collapsing white dwarf. The initial density structure of the envelope is taken from the presupernova mantle of the $3.3 M_{\odot}$ helium star (Nomoto \& Hashimoto 1988). Here the explosion energy is assumed to be $1 \times$ $10^{51} \mathrm{ergs} \mathrm{s}^{-1}$ which leads to the synthesis of $0.15 M_{\odot}{ }^{56} \mathrm{Ni}$ behind the shock wave. The calculated light curve, powered by the decays of ${ }^{56} \mathrm{Ni}$ and ${ }^{56} \mathrm{Co}$, reaches much dimmer maximum than typical SNe Ia and declines much faster because of the smaller ejected mass. The good agreement between the calculated light curve and SN 1991bg suggests that the ejecta mass and the kinetic energy of explosion may at least be similar to this model irrespective of the explosion mechanism. Spectroscopic diagnostics would provide a crucial test of this hypothesis.

A Chandrasekhar mass model for SN 1991bg may also be possible. Müller \& Höflich (1994) pointed out that opacities must be low in SN $1991 \mathrm{bg}$ because of low temperatures due to less ${ }^{56} \mathrm{Ni}$ heating, which could explain the fast light curve of SN $1991 \mathrm{bg}$. This model predicts a very short rise time to maximum. The mass of the ejecta can be better constrained from the light curve decline at later phase, since the decline rate is determined by $\gamma$-ray transparency, i.e., the mass and expansion velocities of the ejecta (Leibundgut \& Pinto 1992).

Ruiz-Lapuente et al. (1993b) noted that the late time spectrum of SN $1991 \mathrm{bg}$ shows evidence of emission from low velocity hydrogen gas. If this is confirmed, the companion star might be a hydrogen-rich star, which would be crucial to identify the companion and the explosion mechanism of at least sub-luminous SNe Ia. 


\section{Variations}

\subsection{Light Curves and ${ }^{56} \mathrm{Ni}$ Mass}

In the thermonuclear explosion of white dwarfs, a large amount of ${ }^{56} \mathrm{Ni}$ is synthesized. In these models, the light curves are powered by the radioactive decays ${ }^{56} \mathrm{Ni} \rightarrow{ }^{56} \mathrm{Co}$ $\rightarrow{ }^{56} \mathrm{Fe}$. Müller \& Höflich (1994) presented the currently most sophisticated light curve models. Figure 9 shows simpler approach to the bolometric light curves of W7 and the carbon detonation model of $M=1.03 M_{\odot}$ (CDT3), respectively, where constant optical opacity, $\kappa=0.1,0.2,0.3 \mathrm{~cm}^{2} \mathrm{~g}^{-1}$, is chosen to reproduce the date of maximum brightness in view of uncertainties of the expansion opacity (Nomoto et al. 1994).

The reported variation of the maximum brightness of SNe Ia (Phillips 1993) may be due to the combined variations of the ${ }^{56} \mathrm{Ni}$ mass and the date of maximum. The amount of ${ }^{56} \mathrm{Ni}$ depends on (i) the flame speed in the fast deflagration models, (ii) the location of the transformation into a detonation in the delayed/late detonation models, and (iii) the white dwarf mass in the sub-Chandrasekhar mass models. Because of variations of these parameters, a large variation of the ${ }^{56} \mathrm{Ni}$ mass may be possible.

However, the amount of ${ }^{56} \mathrm{Ni}$ is closely related to the explosion energy $E$, and therefore is constrained from the abundances and expansion velocities of various elements estimated from the spectra. For the Chandrasekhar mass models, the abundance distribution in the velocity space should be similar to W7 $\left(M_{\mathrm{Ni}}=0.58 M_{\odot}\right.$ and $\left.E=1.3 \times 10^{51} \mathrm{erg}\right)$, so that the ${ }^{56} \mathrm{Ni}$ mass is constrained as $0.6 \pm 0.1 M_{\odot}$ as is also the case for other favored models DD4 (Woosley \& Weaver 1994a) and N32 and PDD3 (Khokhlov 1991b). SubChandrasekhar mass models for $M \approx 1.0-1.1 M_{\odot}$ produce similar amounts of ${ }^{56} \mathrm{Ni}$ and show an abundance structure in the velocity space similar to W7 (Fig. 5).

For the light curve, the above variations of the ${ }^{56} \mathrm{Ni}$ mass predict a different relation between the maximum brightness and the post-maximum decline rate. The light curve shape depends mainly on the effective diffusion time $\tau_{m} \propto\left(\kappa M / v_{\exp } c\right)^{1 / 2}$ with $v_{\text {exp }}$ $\propto\left(M_{\mathrm{Ni}} / M\right)^{1 / 2}$ (Arnett 1982). For longer $\tau_{m}$, the decline of the light curve is slower. First let us assume constant optical opacity $\kappa$. Then the model predictions are as follows: 1) For the Chandrasekhar mass models, $M=M_{\mathrm{Ch}}$ is the same but $M_{\mathrm{Ni}}$ varies. For larger $M_{\mathrm{Ni}}, v_{\mathrm{exp}}$ is higher due to larger nuclear energy release and thus $\tau_{m}$ is shorter. This predicts that brighter $\mathrm{SNe}$ Ia declines faster.

2) For the sub-Chandrasekhar mass models, on the contrary, $M_{\mathrm{Ni}}$ varies approximately in proportion to $M$. Then for larger $M, M_{\mathrm{Ni}}$ is larger and $\tau_{m}$ is longer; in other words, brighter SNe Ia tend to decline more slowly.

The effect of optical opacities must also be important as mentioned for SN $1991 \mathrm{bg}$ (Müller \& Höflich 1994; Höflich et al. 1993). Smaller mass ${ }^{56} \mathrm{Ni}$ means less heating of the surface layer, thus lowering $\kappa$ and $\tau_{m}$. This effect might explain the dimmer-faster relation. However, smaller $\kappa$ results in a shorter rise time to maximum and thus brighter maximum (Fig. 9). In other words, smaller ${ }^{56} \mathrm{Ni}$ may not necessarily mean dimmer maximum unless ${ }^{56} \mathrm{Ni}$ mass is exceedingly small such as SN $1991 \mathrm{bg}$.

The light curve shape and maximum brightness may be affected by the deceleration of the ejecta due to circumstellar materials or an outer envelope in the pulsating model (Khokhlov et al. 1993), though the non-homologous behavior of the pulsating envelope depends on the treatment of outer boundaries ( $§ 3.1)$. With larger deceleration, maximum brightness is reached later and thus dimmer, while the decline of the light curve is slower. Thus this effect enhances the brighter-faster tendency of the Chandrasekhar mass models.

If the brighter-slower relation suggested by Phillips (1993) is the case, it might favor the sub-Chandrasekhar mass models as noted in Nomoto (1982b). It should be noted that, if SN 1991T and SN 1991bg are not included, variation among other SNe Ia are 
rather small (Branch et al. 1993). The decay rate of the light curve is also affected by the location of ${ }^{56} \mathrm{Ni}$ (Canal et al. 1988; Nomoto \& Shigeyama 1991 for SN 1986G).

The interpretation of the maximum brightness $L_{\max }$ of SNe Ia in terms of radioactive decay models has been used to estimate the Hubble constant $H_{0}$ (in units of $\mathrm{km} \mathrm{s}^{-1}$ $\mathrm{Mpc}^{-1}$ hereafter: Arnett et al. 1985). From the well-observed SNe Ia, Branch (1992) derived $L_{\max }=2.06 \pm 0.28 \times 10^{43}\left(H_{0} / 50\right)^{-2} \mathrm{erg} \mathrm{s}^{-1}$. Figure 10 shows the dependence of $H_{0}$ on the date of maximum brightness $t_{\max }$ for W7 with constant $\kappa$, where $t_{\max }$ $=19 \pm 2 \mathrm{~d}$ leads to $H_{0}=73 \pm 10$ (Shigeyama et al. 1992; Yamaoka et al. 1994a). From the comparisons with individual SNe Ia, Müller \& Höflich (1994) obtained $H_{0}=$ $66 \pm 10$ for the delayed detonation models. If we adopt the above range of $H_{0}$ and the Cepheid distance to IC 4182 determined with the HST observation (Sandage et al. 1992), SN $1937 \mathrm{C}$ would be somewhat brighter than typical SNe Ia, though it depends on the extrapolation to maximum. However, Branch et al. (1993) noted that SN 1937C belongs to typical SNe Ia in its spectra and speed class. Further careful study of SN $1937 \mathrm{C}$ is needed.

\subsection{Spectra and Mixing}

Supernova spectra are discussed in detail elsewhere in this volume, so that only a few points are discussed here. Important information from the spectra are the expansion velocities of several elements, which can be compared with the theoretical abundance distribution in the velocity space to diagnose the models. Some SNe Ia, 1990N and $1992 \mathrm{~A}$, show high velocity $\mathrm{Si}\left(v_{\exp }>20,000 \mathrm{~km} \mathrm{~s}^{-1}\right.$; see, however, Wheeler et al. 1993) and $\mathrm{Fe} / \mathrm{Co} / \mathrm{Ni}\left(>15,000 \mathrm{~km} \mathrm{~s}^{-1}\right)$ as well as $\mathrm{O}$ in the wide velocity range from $\approx 10,000$ to 20,000 $\mathrm{km} \mathrm{s}^{-1}$ (Leibundgut et al. 1991a; Jeffery et al. 1992; Mazzali et al. 1993; Kirshner et al. 1993). SN $1991 \mathrm{~T}$ has also $\mathrm{Fe} / \mathrm{Co} / \mathrm{Ni}$ at $>15,000 \mathrm{~km} \mathrm{~s}^{-1}$ and $\mathrm{O}$ in the range $\approx 10,000$ to $20,000 \mathrm{~km} \mathrm{~s}^{-1}$ (Jeffery et al. 1992).

The presence of high velocity $\mathrm{Si}$ and Fe could be due to (i) mixing of $\mathrm{Si}$ and Fe to the outer layers for W7 (Branch et al. 1985), (ii) late/delayed detonation, or (iii) carbon detonation in the sub-Chandrasekhar mass white dwarfs (Shigeyama et al. 1992).

The presence of low velocity $\mathrm{O}$ may provide interesting constraints. Since the production of $\mathrm{Fe} / \mathrm{Co} / \mathrm{Ni}$ implies that the temperature at the burning front becomes high enough ( $\gtrsim 4 \times 10^{9} \mathrm{~K}$ ) to deplete all oxygen (Fig. 1; e.g., Thielemann et al. 1994), the local coexistence of $\mathrm{O}$ and $\mathrm{Fe}$ is difficult to realize. Thus the presence of low velocity $\mathrm{O}$ together with higher velocity $\mathrm{Fe}$ implies mixing of $\mathrm{O}$ and $\mathrm{Fe}$ in the velocity space unless Fe is primordial. Such a mixing could occur by convection during the propagation of the deflagration wave (Livne 1993).

Non-spherical explosions induced by late/delayed detonations (see the next section) could also produce non-spherical abundance distribution, i.e., elemental mixing in the velocity space. From the synthetic spectra compared with the observations, mixing is favored by Branch et al. (1985) but not by Harkness (1991). Asymmetries of the explosion might also be responsible for the significant variations in the time evolution of the expansion velocity of Si (Branch et al. 1988; Barbon et al. 1990). However, Filippenko (1989) and Branch and van den Bergh (1993) noted the presence of correlation between the expansion velocity of $\mathrm{Si}$ and the type of host galaxies, which may not be accounted for with asymmetries. SNe Ia in E and S0 galaxies show significantly faster decrease in the expansion velocity with time; it would be due to the steeper density gradient in the outer layer, which may reflect the difference in the mass and circumstellar matter of the progenitors. (See also van den Bergh and Pazder 1992 for brightness - host galaxy type relation.) 

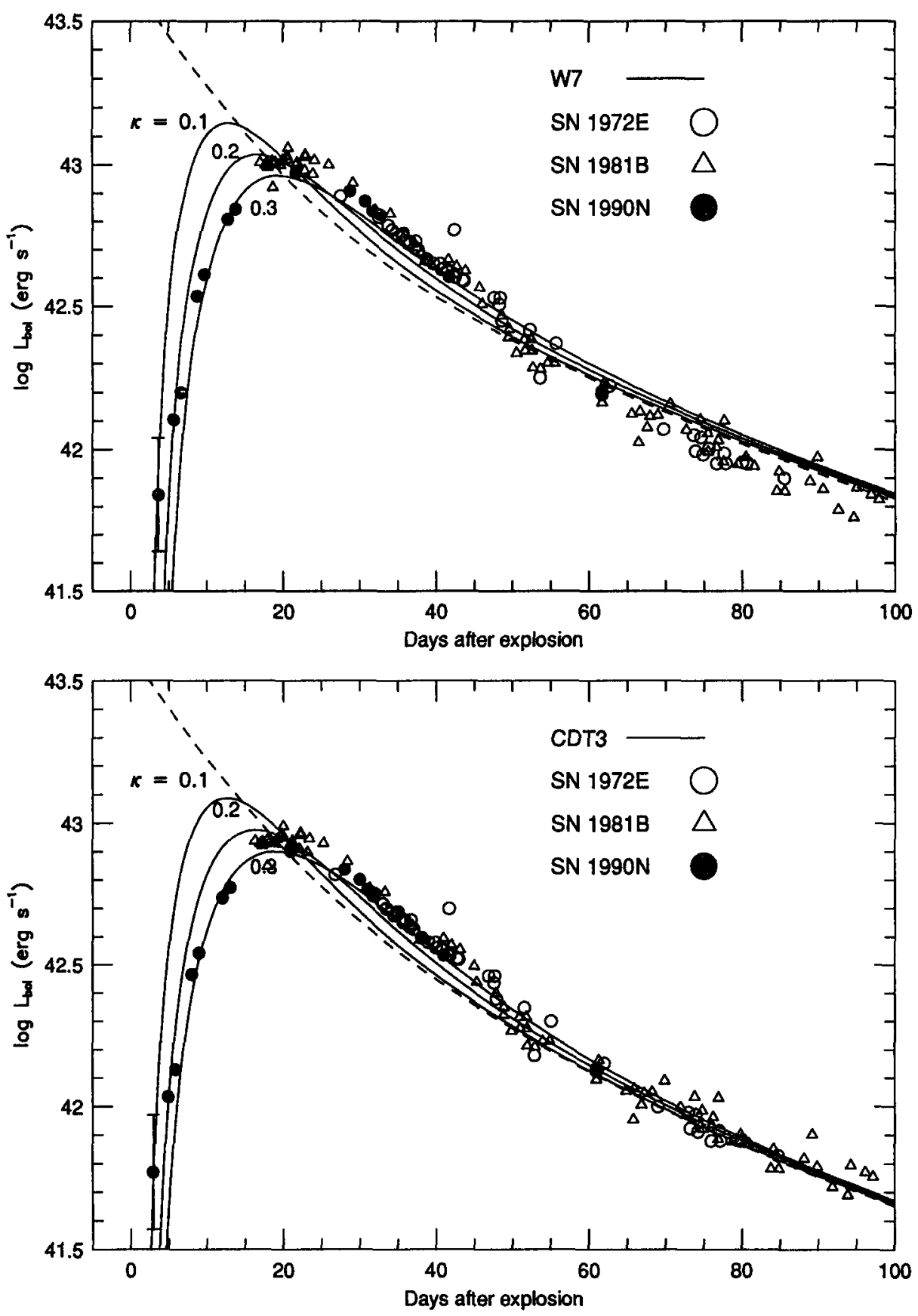

Figure 9. The calculated bolometric light curve for the carbon deflagration model W7 (a) and the carbon detonation model CDT3 (b) for $\kappa=0.1,0.2$, and $0.3 \mathrm{~cm}^{2} \mathrm{~g}^{-1}$ as compared with the observed visual light curves of SN 1972E, 1981B, and 1990N (Leibundgut et al. 1991a,b) (Nomoto 1993). 


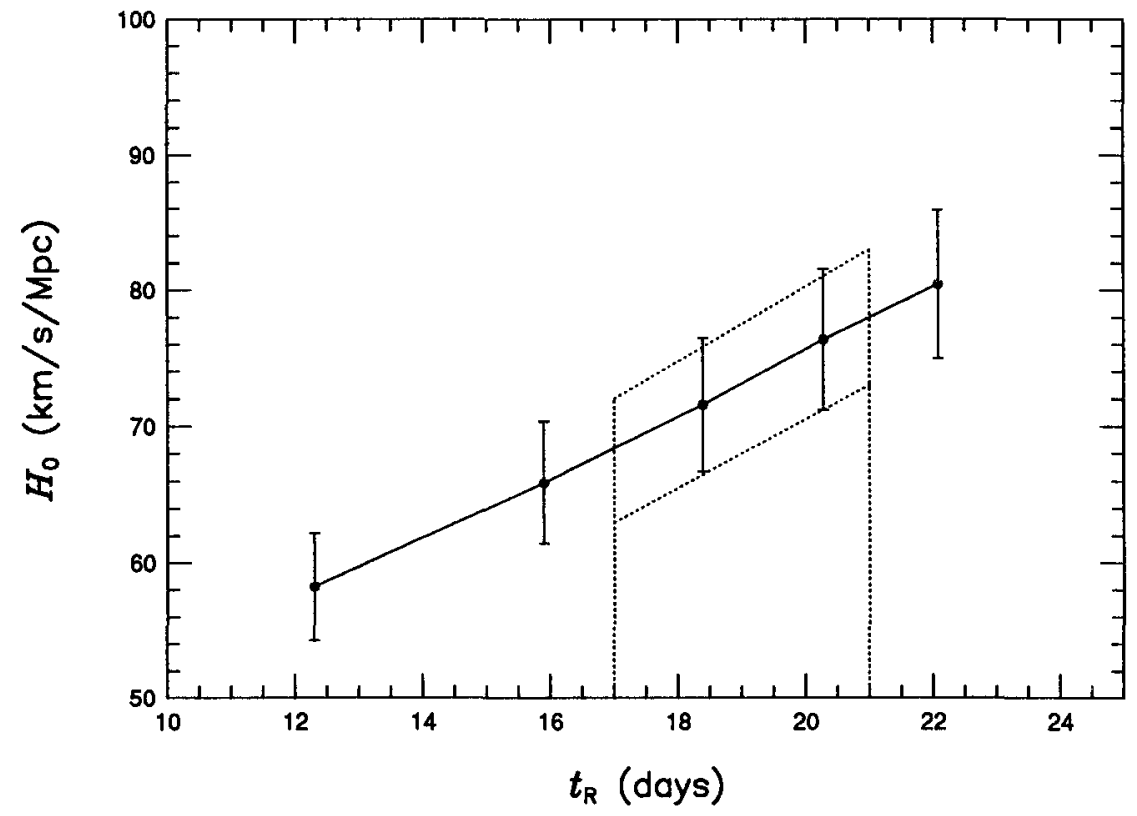

Figure 10. Dependence of the Hubble constant $H_{0}$ on the date of maximum brightness $t_{\max }$ for W7, where $t_{\max }$ is obtained as a function of $\kappa$ (Yamaoka et al. 1994a).

\subsection{Aspherical Explosions and Polarization}

Some theoretical models of SNe Ia predict rather large deviation from spherical explosions. 2D simulations of the deflagration wave have shown the formation of a largely deformed burning front (Livne 1993; Arnett 1994). If the delayed/late detonation are induced by such quite a deformed deflagration, the resultant off-center carbon detonation is likely to start from a point rather than a spherical shell. Such an explosion is likely to form non-spherical ejecta (e.g., Steinmetz et al. 1992). Asymmetries must be a common feature of all the off-center detonation type models, including the He detonation, $\mathrm{He} / \mathrm{C}$ double detonations, and delayed/late detonation. If large asymmetries do exist as predicted by the above models, they should be seen in polarization (Shapiro \& Sutherland 1982; Jeffery 1991). However, spectropolarimetric observations of SN 1992A 2 weeks and 7 weeks after maximum have shown no significant polarization (Fig. 11; Spyromilio \& Bailey 1993), which is an important constraint on the theoretical models.

\section{Isotopic Abundances and Metallicity}

The isotopic ratios of iron peak elements relative to the solar ratios provide important constraints on the models. For the Chandrasekhar mass models, the isotopic ratios are sensitive to the central density at the carbon ignition and metallicity. Overproduction of the sum of ${ }^{54} \mathrm{Fe}$ and ${ }^{58} \mathrm{Ni}$ relative to ${ }^{56} \mathrm{Fe}$ in the deflagration model has been pointed out (Nomoto et al. 1984; Woosley \& Weaver 1986b). These two isotopes originate from mass zones with intermediate neutron excess, which can be caused either by electron capture in the central layer or initial metallicity of the white dwarf in the outer layers where $\mathrm{CNO}$ nuclei were transformed to ${ }^{22} \mathrm{Ne}$ after $\mathrm{H}$ and He burning.

As most SNe Ia occur in spiral galaxies on scale heights larger than 300 pc (Della Valle $\&$ Panagia 1992) where $[\mathrm{Fe} / \mathrm{H}]$ values are 0.0 to -0.5 , one has average metallicities of 


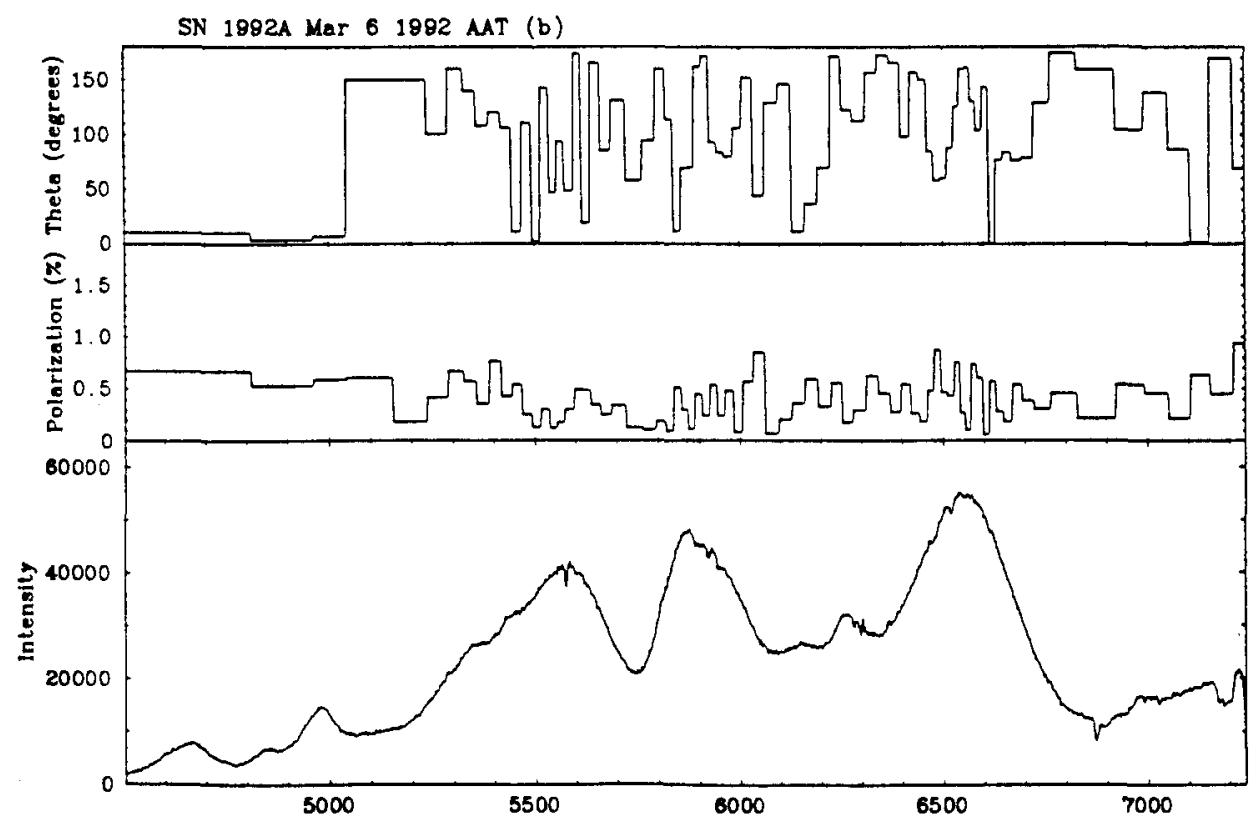

FIGURE 11. Spectropolarimetric observations of SN 1992A 7 weeks after maximum (Spyromilio \& Bailey 1993).

0.5-0.6 times solar. This makes the ${ }^{54} \mathrm{Fe} /{ }^{56} \mathrm{Fe}$ and ${ }^{58} \mathrm{Ni} /{ }^{56} \mathrm{Fe}$ ratios within a factor of 2 and 3 of the solar ratios, respectively (Thielemann et al. 1993), which lie within the present uncertainty range of thermonuclear reaction rates. The chemical separation of ${ }^{22} \mathrm{Ne}$ during the solidification of white dwarfs has the same effects (Isern et al. 1991; Ogata et al. 1993).

The central region of the delayed detonation model undergoes electron capture behind the slower deflagration for a longer time than in W7, thereby having a rather strong constraint on the initial central density of the white dwarf $\left(\lesssim 2 \times 10^{9} \mathrm{~g} \mathrm{~cm}^{-3}\right)$ to avoid overproduction of ${ }^{54} \mathrm{Cr}$ (Khokhlov 1991b). If the carbon ignition density is higher than the threshold for the convective Urca neutrino process due to the Urca pair of ${ }^{23} \mathrm{Na}$ ${ }^{23} \mathrm{Ne}\left(1.8 \times 10^{9} \mathrm{~g} \mathrm{~cm}^{-3}\right)$ and ${ }^{25} \mathrm{Mg}^{25} \mathrm{Ne}\left(1.3 \times 10^{9} \mathrm{~g} \mathrm{~cm}^{-3}\right)$, initiation of the carbon deflagration is delayed to such high density as $\rho_{\mathrm{c}} \gtrsim 4 \times 10^{9} \mathrm{~g} \mathrm{~cm}^{-3}$ (e.g., Iben 1982; Barkat \& Wheeler 1990). Then the overproduction of ${ }^{54} \mathrm{Cr}$ is inevitable in the slow deflagration/delayed detonation models.

The carbon ignition density is higher for larger neutrino cooling during accretion phase. To avoid the convective Urca phase, neutrino emission due to the local Urca shell process of, e.g., ${ }^{21} \mathrm{Ne}-{ }^{21} \mathrm{~F}$ pair (Paczyński 1973) should be suppressed, i.e., the metallicity of accreting white dwarfs should be low enough. For sufficiently low metallicity, the ignition density could be close to the threshold for the convective Urca neutrino process (e.g., Nomoto \& Iben 1985), thus requiring further careful study.

For the sub-Chandrasekhar mass carbon detonation models, since central densities are too low for electron capture to operate, neutron excess in the central region is due only to metallicity (i.e., ${ }^{22} \mathrm{Ne}$ ), thus being much smaller than in the Chandrasekhar mass models. The isotopic ratios of integrated abundances of ejecta after decay of unstable nuclei with respect to solar abundances is shown in Figure 12 for $M=1.05 M_{\odot}$ (Nomoto et al. 


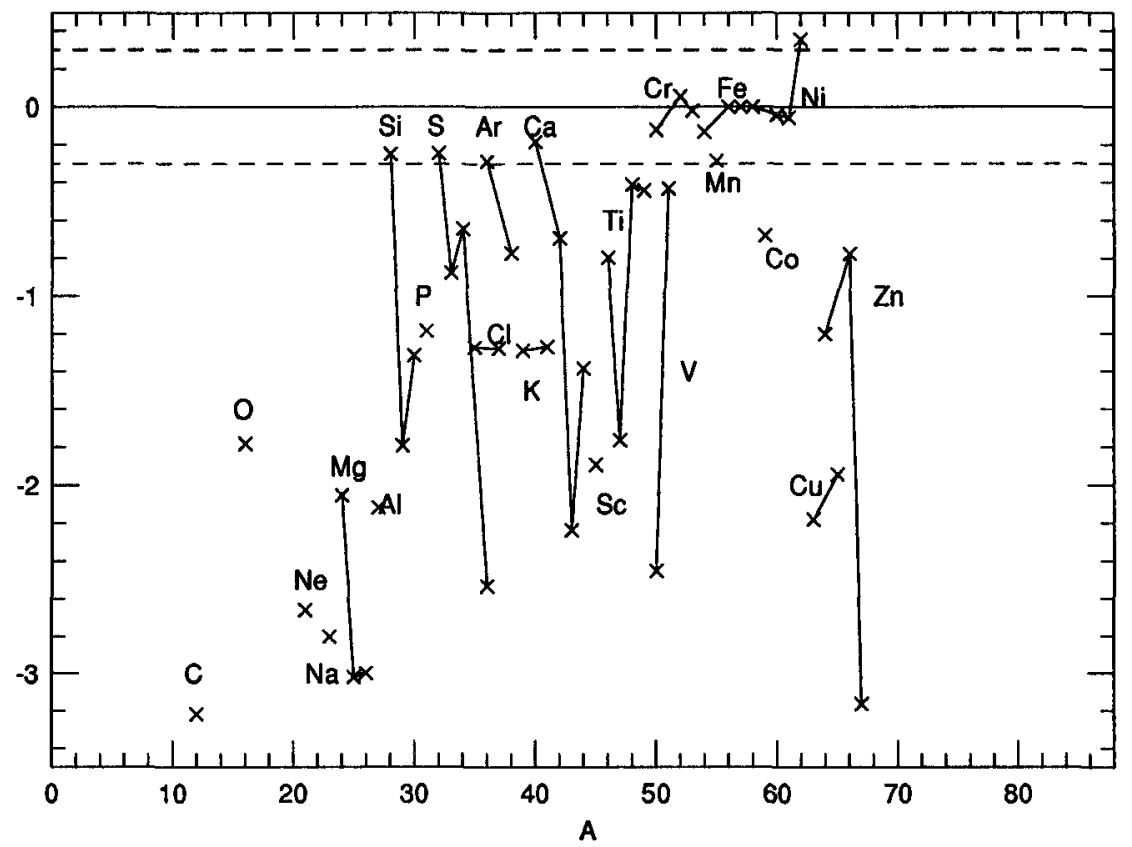

Figure 12. The ratios of integrated abundances of the carbon detonation model with $M=1.05$ $M_{\odot}$ after decay of unstable nuclei, normalized to ${ }^{56} \mathrm{Fe}$, relative to solar abundances (Nomoto et al. 1994).

1994). Overproductions of ${ }^{54} \mathrm{Fe},{ }^{58} \mathrm{Ni}$, and ${ }^{54} \mathrm{Cr}$ are not observed and the isotopic ratios of iron peak elements are within a factor of 2 relative to the solar ratios.

To discuss the degree of overproduction of neutron-rich Fe peak elements, we should taken into account the fact that the observed estimate of SNe Ia frequency is as low as $10 \%$ of the total supernova occurrence so that Fe from SNe Ia is about $50 \%$ of total Fe (van den Bergh \& Tammann 1991; Tsujimoto et al. 1993; Bravo et al. 1993).

\section{Discussion}

Observations have shown significant variations in the light curves and spectra of SNe Ia with the two extreme ends of SN $1991 \mathrm{~T}$ and $1991 \mathrm{bg}$, which have challenged the theoretical models. Possible theoretical explanations of these variations and some critical observations to discriminate the models are as follows. For the Chandrasekhar mass models, there exist some variations in the ignition conditions, such as the central density and temperature of the white dwarf, due to variations of age and metallicity of the white dwarf and the mass accretion rate. This would cause some differences in the flame speed $v_{\text {def }}$, which in turn would yield a significantly different outcome if the transition from deflagration to detonation occurs.

In the late detonation models, if the difference in $v_{\text {def }} / v_{\mathbf{s}}$ is small, differences in the composition in the inner regions and thus the expansion velocity at the outer edge of the inner Fe core are small. However, a large variation of the composition in the outermost layer would result due to late detonation, where nucleosynthesis depends sensitively on the density at this transition. The extremely Fe-rich case would correspond to SN $1991 \mathrm{~T}$. Si-rich cases would be more common, since the transition to detonation may occur more easily at lower densities. Some variations in the expansion velocities of Si may be 
due to the variation of the transition density including the case of no transition (i.e., pure deflagration). For the delayed detonation models including the pulsation cases, the observed variation would also be resulted from the variation of the transition point.

For the sub-Chandrasekhar mass models, variation of the white dwarf mass at the initiation of the carbon detonation may lead to the observed variations. For example, the explosion of a larger white dwarf mass produces larger amount of ${ }^{56} \mathrm{Ni}$ because of the higher densities.

For the light curves, the Chandrasekhar mass models and the sub-Chandrasekhar models predict opposite relationships between the maximum brightness and the decay rate of the bolometric light curve. Therefore, the confirmation of the suggested brighter - slower relation in the observed bolometric light curves is crucial (Phillips 1993). With more samples, it should be clarified whether SN $1991 \mathrm{~T}$ and 1991bg are rather exceptional cases (Branch et al. 1993). If opacity effects are significant, variation of the rise time to maximum brightness $t_{\max }$ is expected to be large. Variation of the late decline rate would be more useful, since the late light curve is determined by $\gamma$-ray transparencies. Early line $\gamma$-ray observations are particularly important to constrain the mass and distribution of ${ }^{56} \mathrm{Ni}$ in SNe Ia (e.g., Burrows et al. 1991; Lichti et al. 1993; Shigeyama et al. 1993; Ruiz-Lapuente et al. 1993a).

Spectra suggest a certain non-spherical abundance distribution, i.e., mixing in the velocity space from low velocity O and high velocity $\mathrm{Fe}$ in $\mathrm{SN} 1990 \mathrm{~N}$ and 1992A; UV observations at early phase are necessary to confirm high velocity Fe and Co (Wheeler \& Harkness 1990; Wheeler et al. 1993). Theoretically the mechanism of mixing in velocity space should be explored, which would require multi-dimensional high resolution calculations of the propagation of the deflagration/detonation.

Sub-Chandrasekhar mass models that invoke the off-center He detonation predict the presence of He together with ${ }^{56} \mathrm{Ni}$ in the outermost layers. Non-LTE spectral calculations are necessary to see whether He features can be hidden. This is important also to understand the nature of SNe Ic and $\mathrm{SNe} \mathrm{Ib}$, i.e., to provide constraint on the amount of He in SNe Ic (Nomoto et al. 1990; Swartz et al. 1993).

Asymmetries in the explosions are expected to be significant for the off-center detonation type models such as the $\mathrm{He} / \mathrm{C}$ detonations in the sub-Chandrasekhar mass models and the delayed/late carbon detonations. Asymmetric expansions might cause the spectral variations in the early phase. Therefore, confirmation of the negligible asymmetry by the polarization observations is crucial (Jeffery 1991; Spyromilio \& Bailey 1993).

\section{Acknowledgements}

We would like to thank M. Hashimoto and F.-K. Thielemann for collaborative work on the subjects discussed in this paper. This work has been supported in part by the grant-in-Aid for Scientific Research (05242102, 05242103, 06233101, 06740181, 4227) of the Ministry of Education, Science, and Culture in Japan.

\section{REFERENCES}

Arnett, W.D. 1969, Ap\&A 5, 180

Arnett, W.D. 1982, ApJ 253, 785

Arnett, W.D. 1994, in this volume

Arnett, W.D., Branch, D., \& Wheeler, J.C. 1985, Nature 314, 337

Barbon, R., Benetti, S., Cappellaro, E., Rosino, L., \& Turatto, M. 1990, A\&A 237, 79 
Barkat, Z., Livne, E., Swartz, D.A., \& Wheeler, J.C. 1990, in Supernovae, ed. J.C. Wheeler et al. (World Scientific), p. 133

Barkat, Z., \& Wheeler, J.C. 1990, ApJ 355, 602

Benz, W., Bowers, R.L., Cameron, A.G.W., \& Press, W. 1990, ApJ 348, 647

Blinnikov, S.I., \& Kohkhlov, A.M. 1986, Sov. Astron. Let., 12, 131

Branch, D., Doggett, J.B., Nomoto, K., \& Thielemann, F.-K. 1985, ApJ 294, 619

Branch, D., Drucker, W., \& Jeffery, D.J. 1988, ApJ 330, L117

Branch, D., Fisher, A., \& Nugent, P. 1993, AJ 106, 2383

Branch, D., Nomoto, K., \& Filippenko, A.V. 1991, Comments on Astrophysics XV, 221

Branch, D., \& Tammann, G. 1992, ARA\&A 30, 359

Branch, D., \& van den Bergh, S. 1993, AJ 105, 2231

Bravo, E., Isern, J., \& Canal, R. 1993, A\&A 269, 187

Burrows, A., Shankar, A., \& Van Riper, K.A. 1991, ApJ 379, L7

Canal, R., Isern, I., \& López, R. 1988, ApJ 330, L113

Canal, R., \& Ruiz-Lapuente, P. 1994, in this volume

Della Valle, M., \& Panagia, N. 1992, AJ 104, 696

Dgani, R., \& Livio, M. 1990, ApJ 361, 540

Filippenko, A.V. 1989, PASP 101, 588

Filippenko, A.V., et al. 1992a, ApJ 384, L15

Filippenko, A.V., et al. 1992b, AJ 104, 1543

Hachisu, I., Eriguchi, Y., \& Nomoto, K. 1986, ApJ 308, 161

Harkness, R.P. 1991, in SN 1987A and Other Supernovae, eds. I.J., Danziger \& K. Kjär (European Southern Observatory, Garching), p.447

Harkness, R.P., \& Wheeler, J.C. 1990, in Supernovae, ed. A. Petschek (Springer, Berlin), p. 1.

Hashimoto, M., Iwamoto, K., \& Nomoto, K. 1993, ApJ 414, L105

Hashimoto, M., Nomoto, K., Arai, K., \& Kaminisi, K. 1986, ApJ 307, 687

Höflich, P., Müller, E., \& Khokhlov, A.M. 1993, A\&A 268, 570

Iben, I.Jr. 1982, ApJ 253, 248

Iben, I.Jr. 1988, ApJ 324, 355

Iben, I.Jr., \& Tutukov, A. 1984, ApJ Suppl. 55, 335

Isern, J., Canal, R., \& Labay, J. 1991, ApJ 372, L83

Ivanova, L.N., Imshennik, V.S., \& Chechetkin, V.M. 1975, Ap\&SS 31, 497

Jeffery, D. 1991, ApJ 375, 264

Jeffery, D., Leibundgut, B., Kirshner, R.P., Benetti, S., Branch, D., \& Sonneborn, G. 1992, ApJ 397,304

Khokhlov, A.M. 1991a, A\&A 245, 114

Khokhlov, A.M. 1991b, A\&A 245, L25

Khokhlov, A.M., Müller, E., \& Höflich, P. 1993, A\&A 270, 23

Kirshner, R.P. et al. 1993, ApJ 415, 589

Leibundgut, B., Kirshner R.P., Filippenko, A.V., Shields, J.C., Foltz, C.B., Phillips, M.M., \& Sonneborn, G. 1991a, ApJ 371, L23

Leibundgut, B., \& Pinto, P.A. 1992, ApJ 401, 49

Leibundgut, B., Tammann, G.A., Cadonau, R., \& Cerrito, D. 1991b, A\&AS 89, 537

Leibundgut, B., et al. 1993, AJ 105, 301

Lichti, G.G. et al. A\&AS 97, 215

Livne, E. 1990, ApJ 354, L53

Livne, E. 1993, ApJ 406, L17 
Lucy, L.B. 1991, ApJ 383, 308

Mazzali, P. 1992, private communication

Mazzali, P., Lucy, L.B., Danziger, I.J., Gouiffes, C., Cappellaro, E.R., \& Turatto, M. 1993, A\&A 269,423

Miyaji, S., Nomoto, K., Yokoi, K., \& Sugimoto, D. 1980, PASJ 32, 303

Mochkovitch, R., \& Livio, M. 1990, A\&A 236, 378

Müller, E., \& Arnett, W.D. 1986, ApJ 307, 619

Müller, E., \& Höflich, P. 1994, A\&A 281, 51

Nomoto, K. 1982a, ApJ 253, 798

Nomoto, K. 1982b, ApJ 257, 780

Nomoto, K. 1982c, in Supernovae: A Survey of Current Research, ed. M.J. Rees and R.J. Stoneham (Dordrecht: Reidel), p. 210

Nomoto, K. 1986, Ann. NY Acad. Sci. 470, 294

Nomoto, K. 1993, in The Realm of Interacting Binary Stars, ed. J. Sahade et al. (Kluwer, ASSL), p. 247

Nomoto, K., Filippenko, A.V., \& Shigeyama, T. 1990, A\&A 240, L1

Nomoto, K., \& Hashimoto, M. 1988, Phys. Rep. 163, 13

Nomoto, K., \& Iben, I.Jr. 1985, ApJ 297, 531

Nomoto, K., \& Kondo, Y. 1991, ApJ 367, L19

Nomoto, K., \& Shigeyama, T. 1991, in Supernovae, ed. Woosley S.E. (Springer, Berlin), p. 572.

Nomoto, K., \& Sugimoto, D. 1977, PASJ 29, 765

Nomoto, K., Sugimoto, D., \& Neo, S. 1976, ApSS 39, L37

Nomoto, K., Thielemann, F.-K., \& Yokoi, K. 1984, ApJ 286, 644

Nomoto, K., Yamaoka, H., Shigeyama, T., Kumagai, S., \& Tsujimoto, T. 1991, in Neutron Stars: Observations and Theory, eds. J. Ventura and D. Pines (Kluwer, NATO ASI) p. 143

Nomoto, K., Yamaoka, H., Shigeyama, T., Kumagai, S., \& Tsujimoto, T. 1994, in Supernovae (Les Houches, Session LIV), ed. S. Bludman et al. (Elsevier Sci. Publ.), p. 199

Ogata, S., Iyetomi, H., \& Ichimaru, S. 1993, Phys. Rev. E 48, 1344

Paczyński, B. 1973, Acta Astr. 23, 1

Phillips, M.M. 1993, ApJ 413, L105

Phillips, M.M., et al. 1992, AJ 103, 1632

Renzini, A. 1994, in this volume

Ruiz-Lapuente, P., Cappellaro, E., Turatto, M., Gouiffes, C., Danziger, I.J., Della Valle, M., \& Lucy, L.B. 1992, ApJ 387, L33

Ruiz-Lapuente, P., Jeffery, D.J., Challis, P.M., Filippenko, A.V., Kirshner, R.P., Ho, L.C., Schmidt, B.P., Sanchez, F., \& Canal, R. 1993b, Nature 365, 728

Ruiz-Lapuente, P., Lichti, G.G., Lehoucq, R., Canal, R., \& Cassé 1993a, ApJ 417, 547

Sandage, A., Saha, A., Tammann, G.A., Panagia, N., \& Macchetto, D. 1992, ApJ 401, L7

Shapiro, P.R., \& Sutherland, P.G. 1982, ApJ 263, 902

Shigeyama, T., Kumagai, S., Yamaoka, H., Nomoto, K., \& Thielemann, F.-K. 1993, A\&AS 97, 223

Shigeyama, T., Nomoto, K., Yamaoka, H., \& Thielemann, F.-K. 1992, ApJ 386, L13

Spyromilio, J. \& Bailey, J.A. 1993, Proc. Astr. Soc. Australia 10, 263

Steinmetz, M., Müller, E., \& Hillebrandt, W. 1992, A\&A 254, 177

Sugimoto, D., \& Nomoto, K. 1980, Space Sci. Rev. 25, 155

Swartz, D.A., Filippenko, A.V., Nomoto, K., \& Wheeler, J.C. 1993, ApJ 411, 313

Takahara, M., Hino, M., Oda, T., Muto, K., Wolters, A.A., Glaudemans, P.W.M., \& Sato, K., 1989, Nucl. Phys. A504, 167 
Thielemann, F.-K., Hashimoto, M., \& Nomoto, K. 1990, ApJ 349, 222

Thielemann, F.-K., Nomoto, K., \& Hashimoto, M. 1993, in The Origin and Evolution of the Elements, ed. N. Prantzos (Cambridge University Press), p. 297

Thielemann, F.-K., Nomoto, K., \& Hashimoto, M. 1994, in Supernovae (Les Houches, Session LIV), ed. S. Bludman et al. (Elsevier Sci. Publ.), p. 629

Thielemann, F.-K., Nomoto, K., \& Yokoi, K. 1986, A\&A 158, 17

Tsujimoto, T., Iwamoto, K., Hashimoto, M., Nomoto, K., \& Thielemann, F.-K., 1993, in Origin and Evolution of the Elements, ed. K. Kubono \& T. Kajino (World Scientific), p. 50

van den Bergh, S., \& Pazder, J. 1992, ApJ 390, 34

van den Bergh, S., \& Tammann, G. 1991, ARA\&A 29, 363

Webbink, R. 1984, ApJ 277, 355

Wheeler, J.C., \& Harkness, R. 1990, Rep. Prog. Phys. 53, 1467

Wheeler, J.C., Swartz, D., \& Harkness, R. 1993, Phys. Rep. 227, 113

Williams, F.A. 1985, Combustion Theory (Menlo Park: Benjamin/Cummings), p. 217

Woosley, S.E. 1990, in Supernovae, ed. A. Petschek (Springer), p. 182

Woosley, S.E., Taam, R.E., \& Weaver, T.A. 1986, ApJ 301, 601

Woosley, S.E., \& Weaver, T.A. 1986a, Lecture Notes in Physics, 255, 91

Woosley, S.E., \& Weaver, T.A. 1986b, ARA\&A 24, 205

Woosley, S.E., \& Weaver, T.A. 1994a, in Supernovae (Les Houches, Session LIV), ed. S. Bludman et al. (Elsevier Sci. Publ.), p. 63

Woosley, S.E., \& Weaver, T.A. 1994b, ApJ, 423, 371

Yamaoka, H., Nomoto, K., Shigeyama, T., \& Hashimoto, M. 1994b, in preparation

Yamaoka, H., Shigeyama, T., \& Nomoto, K. 1994a, in Evolution of the Universe and its Observational Quest, ed. K. Sato (Universal Academy Press, Tokyo), p. 565

Yamaoka, H., Shigeyama, T., Nomoto, K., \& Thielemann, F.-K. 1992, ApJ 393, L55 